\title{
WALKS IN PATH GRAPH ON FOUR VERTICES AND FIBONACCI SEQUENCE
}

\section{RADIM HOŠEK}

Received 05 April, 2016

\begin{abstract}
Using elementary knowledge of graph theory, we show that a path graph on four vertices exhibits Fibonacci structure. For arbitrary start and end vertices, the number of walks of any length is given by a Fibonacci number.
\end{abstract}

2010 Mathematics Subject Classification: 05C38; 05C50; 11B39

Keywords: path graph, walk in graph, Fibonacci sequence, Fibonacci structure

The Fibonacci sequence is a well known mathematical object, having interesting connections to different features in nature. It is given by a simple recurrence formula.

Definition 1. A sequence $\Phi: \mathbb{N} \rightarrow \mathbb{N}$ defined with

$$
\Phi(n+1)=\Phi(n)+\Phi(n-1), \quad \forall n \in \mathbb{N},
$$

with initial values $\Phi(0)=0, \Phi(1)=1$ is called the Fibonacci sequence.

In this short comment we reveal the link between Fibonacci numbers and number of walks in a path graph on four vertices, i.e. of length three, see graph $P_{3}$ in Figure 1. Note that the Fibonacci structure is linked with $P_{3}$ and cannot be generalized to $P_{N}$.

FIGURE 1. Path-graph of length three.

To avoid any confusion, we recall the definition of a walk in a graph, see [2, p. 9]. A walk of length $k$ in graph $G$ is a non-empty alternating sequence $v_{0} e_{0} v_{1} e_{1} \ldots$ $e_{k-1} v_{k}$ of vertices and edges in $G$ such that $e_{i}=\left\{v_{i}, v_{i+1}\right\}$ for all $i<k$.

We denote with

$$
A:=\left(\begin{array}{llll}
0 & 1 & 0 & 0 \\
1 & 0 & 1 & 0 \\
0 & 1 & 0 & 1 \\
0 & 0 & 1 & 0
\end{array}\right),
$$


the adjacency matrix of $P_{3}$. An elementary assertion of graph theory (see [1, Lemma 2.5]) gives the number of walks of length $k$ between vertices $i$ and $j$ as the appropriate element of $k$-th power of the adjacency matrix $B$ of the graph, i.e.

$$
w_{k, i, j}:=\left\{B^{k}\right\}_{i, j}
$$

The following lemma enables us to compute powers of adjacency matrix of a path graph (regardless the number of vertices).

Lemma 1. Let $B \in \mathbb{R}^{N \times N}$ be an adjacency matrix of a path graph of length $N-1$. Then for any $k \in \mathbb{N}$ it holds that

$$
\left\{B^{k+1}\right\}_{i, j}=\left\{B^{k}\right\}_{i-1, j}+\left\{B^{k}\right\}_{i+1, j},
$$

and

$$
\left\{B^{k+1}\right\}_{i, j}=\left\{B^{k}\right\}_{i, j-1}+\left\{B^{k}\right\}_{i, j+1},
$$

where we set $\left\{B^{p}\right\}_{l, m}=0$ for any $p \in \mathbb{N}$ and $(l, m) \notin\{1, \ldots, N\}^{2}$.

Proof. The claim immediately follows from graph theory interpretation of $(0.3)$ : Any walk of length $k+1$ between vertices $i$ and $j$ contains a unique subwalk of length $k$ from either $i-1$ or $i+1$ (if such vertex exists) to $j$. Hence we can conclude (0.4), similar argument proves also (0.5).

Note that any path-graph is a bipartite graph, which implies non-existence of walk of odd length between vertices of the same parity (and walk of even length between vertices of different parity), i.e. $\left\{B^{k}\right\}_{i, j}=0$ if the parity of $k$ and $i+j$ differs. Using this property we define the following split of $k$-th power of the adjacency matrix of $P_{3}$, to matrices of corner, edge and middle elements, as they represent three qualitatively distinct cases.

We set matrices $C_{k}, E_{k}, M_{k}$ for $k \in \mathbb{N}$ with

$$
\begin{aligned}
\left\{C_{k}\right\}_{i, j}: & = \begin{cases}\left\{A^{k}\right\}_{i, j} & (i, j) \in\{1,4\} \times\{1,4\} \text { and }(i+j+k) \text { even } \\
0 & \text { otherwise },\end{cases} \\
\left\{E_{k}\right\}_{i, j} & := \begin{cases}\left\{A^{k}\right\}_{i, j} & (i, j) \in(\{2,3\} \times\{1,4\}) \cup(\{1,4\} \times\{2,3\}) \\
0 & \text { and }(i+j+k) \text { even, }\end{cases} \\
\left\{M_{k}\right\}_{i, j}: & = \begin{cases}\left\{A^{k}\right\}_{i, j} & \text { otherwise, } \\
0 & \text { otherwise. }\end{cases}
\end{aligned}
$$

They create a complete split of $A^{k}$, i.e. $A^{k}=C_{k}+E_{k}+M_{k}$ and maximally one of them has a non-zero contribution for each element $\left\{A^{k}\right\}_{i, j}$, as it is illustrated in the following scheme. 


$$
A^{k}=\left(\begin{array}{cccc}
0 & E_{k} & 0 & C_{k} \\
E_{k} & 0 & M_{k} & 0 \\
0 & M_{k} & 0 & E_{k} \\
C_{k} & 0 & E_{k} & 0
\end{array}\right), \quad A^{l}=\left(\begin{array}{cccc}
C_{l} & 0 & E_{l} & 0 \\
0 & M_{l} & 0 & E_{l} \\
E_{l} & 0 & M_{l} & 0 \\
0 & E_{l} & 0 & C_{l}
\end{array}\right),
$$

for $k=2 s-1, l=2 s, s \in \mathbb{N}$.

We call elements defined by first lines in (0.6), (0.7) or (0.8) structural non-zeros. Note that $C_{1}$ is a zero matrix, the only one whose structural non-zeros attain zero value.

Matrix $C_{k}$ of corner elements represents walks between vertices of the degree 1 (not necessarily distinct), $E_{k}$ represents the walks connecting vertices with different degrees and finally $M_{k}$ represents the walks between vertices with degree equal to 2 .

We use the previously established split in the next Theorem.

Theorem 1. For given $k \in \mathbb{N}$, the structural non-zeros of matrix $C_{k}\left(E_{k}, M_{k}\right)$ are equal to a constant, which we denote by $c_{k}\left(e_{k}, m_{k}\right)$. Moreover, it holds that

$$
c_{k}=\Phi(k-1), \quad e_{k}=\Phi(k), \quad m_{k}=\Phi(k+1) .
$$

Proof. Applying (0.5) to all structural non-zeros of $C_{k+1}$ we get

$$
c_{k+1}=e_{k} .
$$

Applying (0.4) and (0.5) to structural non-zeros of $E_{k+1}$ gives two relations

$$
\begin{gathered}
e_{k+1}=m_{k}, \\
e_{k+1}=c_{k}+e_{k} .
\end{gathered}
$$

Combining (0.10) and (0.12) one gets

$$
c_{k+2}=e_{k+1}=c_{k}+e_{k}=c_{k+1}+c_{k} .
$$

From (0.2) we deduce initial values $c_{1}=0$ and, with the help of (0.10), $c_{2}=e_{1}=1$. Hence we conclude $c_{k}=\Phi(k-1)$ for any $k \in \mathbb{N}$. Then (0.10) and (0.11) imply $e_{k}=\Phi(k)$ and $m_{k}=\Phi(k+1)$, for $k \in \mathbb{N}$.

We can conclude from Theorem 1 , that any non-zero entry in $A^{k}$ is a Fibonacci number. In other words, number of $k$-walks in $P_{3}$ from $i$ to $j$ is represented by a Fibonacci number $\Phi(n)=\Phi(n(i, j, k))$, where $n(i, j, k) \in\{k-1, k, k+1\}$ according to (0.6)-(0.8) and Theorem 1.

Remark 1. The link between Fibonacci sequence and walks in path graph was revealed during the research on a generalization of bistable equation with non-smooth minima, see [3] or [4]. When substituting the double-well potential with a quadruplewell one, then the nontrivial and qualitatively distinct stationary solutions can be 
represented by walks in graph $P_{3}$, provided additional geometrical constraints are met by the potential to ensure the transitions between minima have the equal length.

\section{ACKNOWLEDGEMENT}

The author was supported by the Grant 13-00863S of the Grant Agency of the Czech Republic.

\section{REFERENCES}

[1] N. Biggs, Algebraic graph theory. 2nd ed., 2nd ed. Cambridge: Cambridge University Press, 1994. doi: 10.1017/CBO9780511608704.

[2] R. Diestel, Graph theory. 3rd revised and updated ed., 3rd ed. Berlin: Springer, 2005.

[3] P. Drábek and R. Hošek, "Properties of solution diagrams for bistable equations." Electron. J. Differ. Equ., vol. 2015, no. 156, pp. 1-19, 2015.

[4] P. Drábek, R. F. Manásevich, and P. Takáč, "Manifolds of critical points in a quasilinear model for phase transitions." in Nonlinear elliptic partial differential equations. Workshop in celebration of Jean-Pierre Gossez's 65th birthday, Bruxelles, Belgium, September 2-4, 2009. Providence, RI: American Mathematical Society (AMS), 2011, pp. 95-134.

Author's address

\section{Radim Hošek}

R.H., Department of Mathematics and NTIS, University of West Bohemia, Univerzitní 22, 30614 Plzeň, Czech Republic,

E-mail address: radhost@kma.zcu.cz 